\title{
On an Attempt to Study the Inter-relationship amongst Various Success Factors behind Adoption of ICT and CAD for Carpet Weaving with the Help of ISM and Fuzzy Mic-Mac Methodology
}

\author{
S. P. Singh \\ Department of \\ Management Studies, IIT \\ Delhi
}

\author{
Lakshay Aggarwal \\ Recventure Education \\ Services Private Limited \\ Delhi, India
}

\author{
Viraj Voditel \\ Techture Structures \\ Private Limited \\ Nagpur, India
}

\author{
Remica Aggarwal \\ Recventures Education \\ Services Private Limited \\ Delhi, India
}

\begin{abstract}
Present research discusses the various success factors for the successful adoption of ICT and CAD in carpet weaving. It further discusses the inter-relationship amongst them using ISM methodology.
\end{abstract}

\section{Keywords}

Interpretive Structural Modelling Methodology; Fuzzy MicMac analysis; Carpet and textile weaving; CAD; ICT

\section{INTRODUCTION}

\subsection{Carpet weaving}

The art of weaving is perhaps as old as human civilization. Carpet weaving constitute a timeless facet of the rich cultural heritage of Asian countries . The carpet industry is a decentralized industry (Darbanhosseiniamirkhiz et al. , 2011) and represents the craft which is carried forward from generations to generations. Egypt is recognized as the original home of carpet making. In the post-British period, carpet making was taken up by many industries which flourished in the regions located in Bhadohi, Mirzapur, and Agra in the state of Uttar Pradesh, Jaipur in the state of Rajasthan and in the Kashmir valley ${ }^{1}$. The Mirzapur-Bhadohi region is also known as "carpet- belt" and accounts for about 85 per cent of Indian carpet exports. The Indian hand-made carpet industry is an export-oriented industry and is a significant contributor to the country's rural economy, which helps in providing employment. At international level , Iran, China, India and Nepal are the key players in the global carpet trade. Other significant exporters include Turkey, Afghanistan, and Pakistan. America and Europe are the chief importers $^{1-3}$.

\subsection{ICT and use of CAD for Carpet and \\ Textile weaving}

CAD/CAM stands for Computer Aided Design/Computer Aided Manufacturing. High quality exportable textile products including apparels, furnishing fabrics, upholsteries, blankets, carpets and so on, are made with help of CAD/CAM system, showing higher potentiality of revenue generation from export market . ICT platform helped in reducing design time, enhance product variety and helped in visualization of designs. These software takes care of all aspects of the value chain of the industry with respect to design, production, marketing, and presentation of designs. The ICT platform helped in increasing the interactivity bringing in customer involvement in product design. Lastly, ICT platform enhanced the richness of design by allowing new color combinations. Digitization also helped in the reduction of cycle time and reduced the wastage of raw materials. Finally, the ICT platform enhanced simultaneously interactivity and market access. Following figure shows the benefits of using ICT and $\mathrm{CAD}$ to weavers [3].

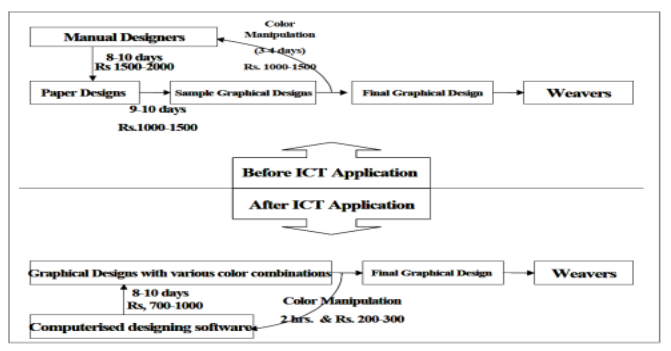

Adapted from Bowonder, B. Swamy, J. and Mastakar, N. (2005)

\subsection{Application of ICT softwares / use of CAD softwares4-6}

Textiles are structurally complex materials and therefore represents the ongoing challenge in the product design and component selection. The component selection involves the selection of material (fibres), yarn types and fabric geometry and construction. CAD systems dedicated to the textile industry offer solutions that not only help in the design stage but also in the manufacturing process, bridging the gap between the design package and the production tool helps to improve the efficiency of and quality of the industrial process. Both the groups i.e. CAD for the artistic design and $\mathrm{CAD}$ for the woven fabric structure have their own importance in relative fields. The aesthetics or artistic designs perspective is of outmost importance for the fabric designers . They use different colour combinations of yarn along with linear density, thread density, weave etc. to get a particular combination. The major modules involved for the fabric appearance include Dobby, Jaquard and Terry . In addition , there are some ancillary modules helping to simulate the fabric, comprising of the yarn, color and weave library . Following table shows the common CAD softwares for woven fabrics, artistic designs . 
Table 1:CAD softwares for woven fabrics and artistic designs

\begin{tabular}{|c|c|}
\hline Software & Module enable of -- \\
\hline $\begin{array}{l}\text { Auto Tex } \\
5.6\end{array}$ & $\begin{array}{c}\text { Creation of Persian, Tibetan, Nepalese or } \\
\text { western designs apart from traditional Indian } \\
\text { designs. }\end{array}$ \\
\hline ColorNet & $\begin{array}{c}\text { selection and creation of color combinations } \\
\text { by buyers, }\end{array}$ \\
\hline AutoShow & $\begin{array}{l}\text { visualization and presentation tool for } \\
\text { designers, manufacturers, and end users. }\end{array}$ \\
\hline DyeTuft & $\begin{array}{l}\text { accurate calculation of wool requirements for } \\
\text { designs with their respective size so as to } \\
\text { reduce wool wastage. }\end{array}$ \\
\hline AutoFlo & $\begin{array}{l}\text { catalogues the designs/products and helps } \\
\text { designers in management and marketing. }\end{array}$ \\
\hline Taleem & $\begin{array}{l}\text { support to generate Taleem, the weaving } \\
\text { language which is used for maintaining } \\
\text { secrecy of new designs. }\end{array}$ \\
\hline Colorways & $\begin{array}{l}\text { Creation of a combination of colours and } \\
\text { giving a wide range of options to buyers. }\end{array}$ \\
\hline Nedgraphics & $\begin{array}{l}\text { Dobby / Jacquard / Textile designer / 2Dor 3D } \\
\text { geometry }\end{array}$ \\
\hline Point Carre & $\begin{array}{c}\text { Dobby / Jacquard / Textile designer dobby / } \\
\text { Yarn / 2D or 3D geometry }\end{array}$ \\
\hline Scotweave & Dobby \\
\hline $\begin{array}{l}\text { Weave } \\
\text { maker }\end{array}$ & Dobby \\
\hline Weave point & Dobby \\
\hline Arhane & Dobby / Jacquard \\
\hline Penelope & Dobby/ Jacquard/ Terry \\
\hline
\end{tabular}

\section{SUCCESS FACTORS AND FACILITATORS FOR ICT ADOPTION}

The ICT platform endowed a variety of benefits and transformed a number of business practices. The main benefits of use of the platform are:

\subsection{Reduction in design time (RDT)}

2.2 Flexibility to increase colour combinations (FCC)

\subsection{Reduction in wastage of material (RWM)}

2.4 Interactivity to perform collaborative design (ICD) involving both designers and users

2.5 Visualization of design prior to manufacturing (VDPM)

2.6 Ability to carry-out online design(ACOD)

2.7 Increasing the number of designs (IND) and the ability to use other forms of styling

2.8 Enhancing the market (EMOA) through online access.

\section{The main facilitators of the ICT adoption have been}

2.9 Entrepreneurial motivation (EM) of the software provider and his ability to provide a comprehensive platform

2.10Training and interaction(T\&I) helped in enhancing the communication of the grass root workers through a local society

2.11Close interaction between the software provider and the exporters (CISP).

2.12 Skill enhancement and Training (SET) to traditional designers enhanced and enriched the design.

2.13 Knowledge management (KM) : The knowledge management can also help in solving the technical queries of weavers and assist them in improving his productivity.

2.14 Web Enabled selling (WES): Web enabled selling has helped the weavers to host their product on the Internet and gain access to the newer and untapped markets.

\section{ISM and FUZZY MIC-MAC METHODOLOGY}

\subsection{ISM based MIC-MAC ANALYSIS MODEL}

Proposed by Warfield in 1974 , ISM methodology is a technique for establishing inter-relationships amongst the criteria of interest. The process begins with the identification of relevant elements and thereafter establishing contextual relationship amongst them . After that the structural self interaction matrix (SSIM) is created using the VAXO concept which is then followed by the creation of initial reachability matrix (IRM). Final reachability matrix (FRM) is then created after correcting IRM for any possibility of transitivity. From the FRM, the reachability set and antecedent set for each criterion is found. Then the intersection of these sets is derived for all elements. The element for which the reachability and intersection sets are the same is the top-level element. Then the reachability matrix is converted into the canonical matrix format by arranging the elements according to their levels. Based on the relative driving power and dependence power, factors are classified in various categories like autonomous, dependent, driver and linkage. Finally, a diagraph is constructed from the canonical matrix .

\subsubsection{Structural Self Interaction matrix (SSIM)}

In this section we will develop the ISM model for the various success factors behind successful adoption of ICT / CAD methodology in carpet and textile weaving. As mentioned in section 2 , about 14 success factors have been studied for exploring the possible inter-relationships using ISM methodology .

\subsubsection{Initial reachability matrix}

The SSIM has been converted in to a binary matrix, called the initial reachability matrix by substituting $\mathrm{V}, \mathrm{A}, \mathrm{X}, \mathrm{O}$ by 1 or 0 as per the case as shown in table II . After incorporating the transitivity, the final reachability matrix is shown below in the table III.

\subsubsection{Final reachability matrix}




\begin{tabular}{|c|c|c|c|c|c|c|c|c|c|c|c|c|c|c|c|}
\hline & & 1 & 2 & 3 & 4 & 5 & 6 & 7 & 8 & 9 & 10 & 11 & 12 & 13 & 14 \\
\hline & & RDT & FCC & $\begin{array}{c}\mathbf{R W} \\
\mathbf{M}\end{array}$ & IDU & $\begin{array}{c}\text { VDP } \\
\mathbf{M}\end{array}$ & $\begin{array}{c}\mathbf{A C O} \\
\mathbf{D}\end{array}$ & IND & $\begin{array}{l}\text { EM } \\
\text { OA }\end{array}$ & EM & T\&I & CISP & SET & KM & WES \\
\hline 1 & RDT & & $\mathrm{V}$ & $\mathrm{V}$ & $\mathrm{X}$ & A & $\mathrm{X}$ & $\mathrm{X}$ & $\mathrm{V}$ & $\mathrm{A}$ & A & $\mathrm{O}$ & $\mathrm{A}$ & $\mathrm{A}$ & $\mathrm{A}$ \\
\hline 2 & FCC & & & $\mathrm{O}$ & A & A & A & A & V & $\mathrm{O}$ & A & $\mathrm{O}$ & A & A & A \\
\hline 3 & RWM & & & & A & A & A & A & A & $\mathrm{O}$ & A & $\mathrm{O}$ & A & A & $\mathrm{O}$ \\
\hline 4 & IDU & & & & & V & V & V & V & A & A & $\mathrm{O}$ & A & A & $\mathrm{O}$ \\
\hline 5 & VDPM & & & & & & V & V & V & A & A & A & A & A & $\mathrm{O}$ \\
\hline 6 & ACOD & & & & & & & $\mathrm{X}$ & A & $\mathrm{O}$ & A & $\mathrm{O}$ & A & $\mathrm{A}$ & A \\
\hline 7 & IND & & & & & & & & A & $\mathrm{O}$ & A & $\mathrm{O}$ & A & A & V \\
\hline 8 & EMOA & & & & & & & & & $\mathrm{O}$ & A & $\mathrm{O}$ & A & A & V \\
\hline 9 & EM & & & & & & & & & & $\mathrm{O}$ & $\mathrm{O}$ & $\mathrm{V}$ & $\mathrm{O}$ & V \\
\hline 10 & T\&I & & & & & & & & & & & $\mathrm{O}$ & $X$ & $\mathrm{~A}$ & $\mathrm{O}$ \\
\hline 11 & CISP & & & & & & & & & & & & $\mathrm{V}$ & $\mathrm{O}$ & $\mathrm{V}$ \\
\hline 12 & SET & & & & & & & & & & & & & $\mathrm{A}$ & $\mathrm{O}$ \\
\hline 13 & $\mathrm{KM}$ & & & & & & & & & & & & & & $\mathrm{O}$ \\
\hline 14 & WES & & & & & & & & & & & & & & \\
\hline
\end{tabular}

Fig 1: Construction of Structural Self- Interaction Matrix

\begin{tabular}{|c|c|c|c|c|c|c|c|c|c|c|c|c|c|c|c|}
\hline & & 1 & 2 & 3 & 4 & 5 & 6 & 7 & 8 & 9 & 10 & 11 & 12 & 13 & 14 \\
\hline & & RDT & FCC & $\begin{array}{c}\mathbf{R W} \\
\mathbf{M}\end{array}$ & IDU & $\begin{array}{c}\text { VDP } \\
\text { M }\end{array}$ & $\begin{array}{c}\mathbf{A C O} \\
\mathbf{D}\end{array}$ & IND & $\begin{array}{l}\text { EM } \\
\text { OA }\end{array}$ & EM & T\&I & CISP & SET & KM & WES \\
\hline 1 & RDT & 1 & 1 & 1 & 1 & 0 & 1 & 1 & 1 & 0 & 0 & 0 & 0 & 0 & 0 \\
\hline 2 & FCC & 0 & 1 & 0 & 0 & 0 & 0 & 0 & 1 & 0 & 0 & 0 & 0 & 0 & 0 \\
\hline 3 & RWM & 0 & 0 & 1 & 0 & 0 & 0 & 0 & 0 & 0 & 0 & 0 & 0 & 0 & 0 \\
\hline 4 & IDU & 1 & 1 & 1 & 1 & 1 & 1 & 1 & 1 & 0 & 0 & 0 & 0 & 0 & 0 \\
\hline 5 & VDPM & 1 & 1 & 1 & 0 & 1 & 1 & 1 & 1 & 0 & 0 & 0 & 0 & 0 & 0 \\
\hline 6 & ACOD & 1 & 1 & 1 & 0 & 0 & 1 & 1 & 0 & 0 & 0 & 0 & 0 & 0 & 0 \\
\hline 7 & IND & 1 & 1 & 1 & 0 & 0 & 1 & 1 & 0 & 0 & 0 & 0 & 0 & 0 & 1 \\
\hline 8 & EMOA & 0 & 0 & 1 & 0 & 0 & 1 & 0 & 1 & 0 & 0 & 0 & 0 & 0 & 1 \\
\hline 9 & EM & 1 & 0 & 0 & 1 & 1 & 0 & 0 & 0 & 1 & 0 & 0 & 1 & 0 & 1 \\
\hline 10 & T\&I & 1 & 1 & 1 & 1 & 1 & 1 & 0 & 1 & 0 & 1 & 0 & 1 & 0 & 0 \\
\hline 11 & CISP & 0 & 0 & 0 & 1 & 1 & 0 & 0 & 0 & 0 & 0 & 1 & 1 & 0 & 1 \\
\hline 12 & SET & 1 & 1 & 1 & 1 & 1 & 1 & 0 & 1 & 0 & 1 & 0 & 1 & 0 & 0 \\
\hline 13 & KM & 1 & 1 & 1 & 1 & 1 & 1 & 0 & 1 & 0 & 1 & 0 & 1 & 1 & 0 \\
\hline 14 & WES & 1 & 1 & 1 & 0 & 0 & 1 & 0 & 0 & 0 & 0 & 0 & 0 & 0 & 1 \\
\hline
\end{tabular}

Fig 2: Initial reachability matrix

\begin{tabular}{|c|c|c|c|c|c|c|c|c|c|c|c|c|c|c|c|c|}
\hline & & 1 & 2 & 3 & 4 & 5 & 6 & 7 & 8 & 9 & 10 & 11 & 12 & 13 & 14 & \\
\hline & & RDT & FCC & $\begin{array}{c}\mathbf{R W} \\
\mathbf{M}\end{array}$ & IDU & $\begin{array}{c}\text { VDP } \\
\text { M }\end{array}$ & $\begin{array}{c}\text { ACO } \\
\text { D }\end{array}$ & IND & $\begin{array}{c}\text { EM } \\
\text { OA }\end{array}$ & EM & T\&I & CISP & SET & KM & WES & D.P \\
\hline 2 & FCC & 0 & 1 & 1 & 0 & 0 & 1 & 0 & 1 & 0 & 0 & 0 & 0 & 0 & 1 & 4 \\
\hline 3 & RWM & 0 & 0 & 1 & 0 & 0 & 0 & 0 & 0 & 0 & 0 & 0 & 0 & 0 & 0 & 2 \\
\hline
\end{tabular}




\begin{tabular}{|c|c|c|c|c|c|c|c|c|c|c|c|c|c|c|c|c|}
\hline 4 & IDU & 1 & 1 & 1 & 1 & 1 & 1 & 1 & 1 & 0 & 0 & 0 & 0 & 0 & 1 & 9 \\
\hline 5 & VDPM & 1 & 1 & 1 & 0 & 1 & 1 & 1 & 1 & 0 & 0 & 0 & 0 & 0 & 1 & 7 \\
\hline 6 & ACOD & 1 & 1 & 1 & 0 & 0 & 1 & 1 & 1 & 0 & 0 & 0 & 0 & 0 & 0 & 6 \\
\hline 7 & IND & 1 & 1 & 1 & 0 & 0 & 1 & 1 & 1 & 0 & 0 & 0 & 0 & 0 & 1 & 4 \\
\hline 8 & EMOA & 1 & 1 & 1 & 0 & 0 & 1 & 1 & 1 & 0 & 0 & 0 & 0 & 0 & 1 & 7 \\
\hline 9 & EM & 1 & 1 & 1 & 1 & 1 & 1 & 1 & 1 & 1 & 0 & 0 & 1 & 0 & 1 & 8 \\
\hline 10 & T\&I & 1 & 1 & 1 & 1 & 1 & 1 & 1 & 1 & 0 & 1 & 0 & 1 & 0 & 1 & 10 \\
\hline 11 & CISP & 1 & 1 & 1 & 1 & 1 & 1 & 1 & 1 & 0 & 0 & 1 & 1 & 0 & 1 & 9 \\
\hline 12 & SET & 1 & 1 & 1 & 1 & 1 & 1 & 1 & 1 & 0 & 1 & 0 & 1 & 0 & 1 & 11 \\
\hline 13 & $\mathrm{KM}$ & 1 & 1 & 1 & 1 & 1 & 1 & 1 & 1 & 0 & 1 & 0 & 1 & 1 & 1 & 12 \\
\hline 14 & WES & 1 & 1 & 1 & 0 & 0 & 1 & 0 & 1 & 0 & 0 & 0 & 0 & 0 & 1 & 4 \\
\hline & De.P & 12 & 13 & 14 & 7 & 8 & 13 & 11 & 13 & 1 & 1 & 1 & 5 & 1 & 11 & \\
\hline
\end{tabular}

\section{Fig 3 : Final reachability matrix}

D.P : Driving power ; De.P : dependence power

\subsection{Level Partition}

From the final reachability matrix, reachability and final antecedent set for each factor are found. The element for which the reachability and intersection sets are same are the top-level element in the ISM hierarchy. After the identification of top level element, it is separated out from the other elements and the process continues for next level of elements. Reachability set, antecedent set, intersection set along with different level for elements have been shown below in table 4.3.1 to table 4.3.9.

Table 4.3.1: Iteration I

\begin{tabular}{|c|c|c|c|c|}
\hline $\begin{array}{l}\text { S. } \\
\text { No. }\end{array}$ & $\begin{array}{c}\text { Reachability } \\
\text { set }\end{array}$ & Antecedent set & $\begin{array}{c}\text { Intersectio } \\
\text { n set }\end{array}$ & Level \\
\hline 1 & 3 & $\begin{array}{c}1,2,3,4,5,6,7,8,9 \\
10,11,12,13,14\end{array}$ & 3 & \multirow{11}{*}{ I } \\
\hline 2 & $2,3,6,8$ & $\begin{array}{l}1,2,4,5,6,7,8,9, \\
10,11,12,13,14\end{array}$ & $2,6,8$ & \\
\hline 3 & $1,2,3,6,8$ & $\begin{array}{c}1,4,5,6,7,8,9,10,1 \\
1,12,13,14\end{array}$ & $1,6,8$ & \\
\hline 4 & $1,2,3,6,8,14$ & $\begin{array}{c}4,5,7,8,9,10,11,1 \\
2,13,14\end{array}$ & 8,14 & \\
\hline 5 & $\begin{array}{c}1,2,3,6,8,7,1 \\
4\end{array}$ & $\begin{array}{c}4,5,7,8,9,10,11,1 \\
2,13\end{array}$ & 7 & \\
\hline 6 & $\begin{array}{c}1,2,3,5,6,7,8 \\
14\end{array}$ & $4,5,9,10,11,12,13$ & 5 & \\
\hline 7 & $\begin{array}{c}1,2,3,4,5,6,7 \\
8,14\end{array}$ & $4,5,9,10,11,12,13$ & 4,5 & \\
\hline 8 & $\begin{array}{c}1,2,3,4,5,6,7 \\
8,12,14\end{array}$ & $9,10,11,12,13$ & 12 & \\
\hline 9 & $\begin{array}{c}1,2,3,4,5,6,7 \\
8,9,12,14\end{array}$ & 9 & 9 & \\
\hline 10 & $\begin{array}{c}1,2,3,4,5,6,7 \\
8,10,12,14\end{array}$ & 10 & 10 & \\
\hline 11 & $\begin{array}{c}1,2,3,4,5,6,7 \\
8,11,12,14\end{array}$ & 11 & 11 & \\
\hline
\end{tabular}

\begin{tabular}{|c|c|c|c|c|}
\hline 12 & $1,2,3,4,5,6,7$, & 13 & 13 & \\
& $8,13,12,14$ & & & \\
\hline
\end{tabular}

Table 4.3.2: Iteration II

\begin{tabular}{|c|c|c|c|c|}
\hline $\begin{array}{l}\text { S. } \\
\text { No. }\end{array}$ & $\begin{array}{c}\text { Reachability } \\
\text { set }\end{array}$ & Antecedent set & $\begin{array}{c}\text { Intersectio } \\
\text { n set }\end{array}$ & Level \\
\hline 2 & $2,6,8$ & $\begin{array}{c}1,2,4,5,6,7,8,9,10 \\
, 11,12,13,14\end{array}$ & $2,6,8$ & \\
\hline 3 & $1,2,6,8$ & $\begin{array}{c}1,4,5,6,7,8,9,10,1 \\
1,12,13,14\end{array}$ & $1,6,8$ & \\
\hline 4 & $1,2,6,8,14$ & $\begin{array}{c}4,5,7,8,9,10,11,1 \\
2,13,14\end{array}$ & 8,14 & \\
\hline 5 & $1,2,6,8,7,14$ & $\begin{array}{c}4,5,7,8,9,10,11,1 \\
2,13\end{array}$ & 7 & \\
\hline 6 & $\begin{array}{c}1,2,5,6,7,8,1 \\
4\end{array}$ & $\begin{array}{c}4,5,9,10,11,12 \\
13\end{array}$ & 5 & II \\
\hline 7 & $\begin{array}{c}1,2,4,5,6,7,8 \\
14\end{array}$ & $\begin{array}{c}4,5,9,10,11,12 \\
13\end{array}$ & 4,5 & \\
\hline 8 & $\begin{array}{c}1,2,4,5,6,7,8 \\
12,14\end{array}$ & $9,10,11,12,13$ & 12 & \\
\hline 9 & $\begin{array}{c}1,2,4,5,6,7,8 \\
9,12,14\end{array}$ & 9 & 9 & \\
\hline 10 & $\begin{array}{c}1,2,4,5,6,7,8 \\
10,12,14\end{array}$ & 10 & 10 & \\
\hline 11 & $\begin{array}{c}1,2,4,5,6,7,8 \\
11,12,14\end{array}$ & 11 & 11 & \\
\hline 12 & $\begin{array}{c}1,2,4,5,6,7,8 \\
13,12,14\end{array}$ & 13 & 3 & \\
\hline
\end{tabular}


Table 4.3.3: Iteration III

\begin{tabular}{|c|c|c|c|c|}
\hline $\begin{array}{l}\text { S. } \\
\text { No. }\end{array}$ & $\begin{array}{c}\text { Reachability } \\
\text { set }\end{array}$ & Antecedent set & $\begin{array}{c}\text { Intersectio } \\
\text { n set }\end{array}$ & Level \\
\hline 2 & $2,6,8$ & $\begin{array}{l}1,2,4,5,6,7,8,9, \\
10,11,12,13,14\end{array}$ & $2,6,8$ & \\
\hline 3 & $1,2,6,8$ & $\begin{array}{c}1,4,5,6,7,8,9,10,1 \\
1,12,13,14\end{array}$ & $1,6,8$ & \\
\hline 4 & $1,2,6,8,14$ & $\begin{array}{c}4,5,7,8,9,10,11,1 \\
2,13,14\end{array}$ & 8,14 & \\
\hline 5 & $1,2,6,8,7,14$ & $\begin{array}{c}4,5,7,8,9,10,11,1 \\
2,13\end{array}$ & 7 & \\
\hline 6 & $\begin{array}{c}1,2,5,6,7,8,1 \\
4\end{array}$ & $\begin{array}{c}4,5,9,10,11,12 \\
13\end{array}$ & 5 & \\
\hline 7 & $\begin{array}{c}1,2,4,5,6,7,8 \\
14\end{array}$ & $\begin{array}{c}4,5,9,10,11,12 \\
13\end{array}$ & 4,5 & \\
\hline 8 & $\begin{array}{c}1,2,4,5,6,7,8 \\
12,14\end{array}$ & $9,10,11,12,13$ & 12 & III \\
\hline 9 & $\begin{array}{c}1,2,4,5,6,7,8 \\
9,12,14\end{array}$ & 9 & 9 & \\
\hline 10 & $\begin{array}{c}1,2,4,5,6,7,8 \\
10,12,14\end{array}$ & 10 & 10 & \\
\hline 11 & $\begin{array}{c}1,2,4,5,6,7,8 \\
11,12,14\end{array}$ & 11 & 11 & \\
\hline 12 & $\begin{array}{c}1,2,4,5,6,7,8 \\
13,12,14\end{array}$ & 13 & 3 & \\
\hline
\end{tabular}

Table 4.3.3: Iteration IV

\begin{tabular}{|c|c|c|c|c|}
\hline $\begin{array}{l}\text { S. } \\
\text { No }\end{array}$ & $\begin{array}{c}\text { Reachability } \\
\text { set }\end{array}$ & Antecedent set & $\begin{array}{c}\text { Intersectio } \\
\text { n set }\end{array}$ & Level \\
\hline 4 & 14 & $\begin{array}{c}4,5,7,9,10,11 \\
12,13,14\end{array}$ & 14 & \multirow{9}{*}{ IV } \\
\hline 5 & 7,14 & $\begin{array}{c}4,5,7,9,10,11 \\
12,13\end{array}$ & 7 & \\
\hline 6 & $5,7,14$ & $\begin{array}{c}4,5,9,10,11,12, \\
13\end{array}$ & 5 & \\
\hline 7 & $4,5,7,14$ & $\begin{array}{c}4,5,9,10,11,12, \\
13\end{array}$ & 4,5 & \\
\hline 8 & $4,5,7,12,14$ & $9,10,11,12,13$ & 12 & \\
\hline 9 & $4,5,7,9,12,14$ & 9 & 9 & \\
\hline 10 & $4,5,7,10,12,14$ & 10 & 10 & \\
\hline 11 & $4,5,7,11,12,14$ & 11 & 11 & \\
\hline 12 & $4,5,7,13,12,14$ & 13 & 3 & \\
\hline
\end{tabular}

Table 4.3.5: Iteration $\mathrm{V}$

\begin{tabular}{|c|c|c|c|c|}
\hline S.No. & $\begin{array}{c}\text { Reachabili } \\
\text { ty set }\end{array}$ & Antecedent set & $\begin{array}{c}\text { Intersec } \\
\text { tion set }\end{array}$ & Level \\
\hline 5 & $\mathbf{7}$ & $4,5,7,9,10,11,12$, & 7 & \\
\hline
\end{tabular}

\begin{tabular}{|c|c|c|c|c|}
\hline & & 13 & & \multirow{8}{*}{$\mathbf{V}$} \\
\hline 6 & 5,7 & $\begin{array}{c}4,5,9,10,11,12 \\
13\end{array}$ & 5 & \\
\hline 7 & $4,5,7$ & $\begin{array}{c}4,5,9,10,11,12 \\
13\end{array}$ & 4,5 & \\
\hline 8 & $4,5,7,12$ & $9,10,11,12,13$ & 12 & \\
\hline 9 & $4,5,7,9,12$ & 9 & 9 & \\
\hline 10 & $\begin{array}{c}4,5,7,10 \\
12\end{array}$ & 10 & 10 & \\
\hline 11 & $\begin{array}{c}4,5,7,11 \\
12\end{array}$ & 11 & 11 & \\
\hline 12 & $\begin{array}{c}4,5,7,13 \\
12\end{array}$ & 13 & 3 & \\
\hline
\end{tabular}

Table 4.3.6: Iteration VI

\begin{tabular}{|c|c|c|c|c|}
\hline S.No. & $\begin{array}{l}\text { Reachabili } \\
\text { ty set }\end{array}$ & Antecedent set & $\begin{array}{c}\text { Intersec } \\
\text { tion set }\end{array}$ & Level \\
\hline 6 & 5 & $4,5,9,10,11,12,13$ & 5 & \multirow{7}{*}{ VI } \\
\hline 7 & 4,5 & $4,5,9,10,11,12,13$ & 4,5 & \\
\hline 8 & $4,5,12$ & $9,10,11,12,13$ & 12 & \\
\hline 9 & $4,5,9,12$ & 9 & 9 & \\
\hline 10 & $4,5,10,12$ & 10 & 10 & \\
\hline 11 & $4,5,11,12$ & 11 & 11 & \\
\hline 12 & $4,5,13,12$ & 13 & 3 & \\
\hline
\end{tabular}

Table 4.3.7: Iteration VII

\begin{tabular}{|c|c|c|c|c|}
\hline S.No. & $\begin{array}{c}\text { Reachabili } \\
\text { ty set }\end{array}$ & Antecedent set & $\begin{array}{c}\text { Intersec } \\
\text { tion set }\end{array}$ & Level \\
\hline 8 & $\mathbf{1 2}$ & $9,10,11,12,13$ & 12 & \\
\hline 9 & 9,12 & 9 & 9 & \multirow{2}{*}{ VII } \\
\cline { 1 - 4 } 10 & 10,12 & 10 & 10 & \multirow{2}{*}{11} \\
\cline { 1 - 4 } 11 & 11,12 & 11 & 3 & \\
\hline 12 & 13,12 & 13 & \multicolumn{2}{|c|}{} \\
\hline
\end{tabular}

Table 4.3.8: Iteration VIII

\begin{tabular}{|c|c|c|c|c|}
\hline S.No. & $\begin{array}{c}\text { Reachabili } \\
\text { ty set }\end{array}$ & Antecedent set & $\begin{array}{c}\text { Intersec } \\
\text { tion set }\end{array}$ & Level \\
\hline 9 & $\mathbf{9}$ & 9 & 9 & \\
\hline 10 & $\mathbf{1 0}$ & 10 & 10 & \multirow{2}{*}{ VIII } \\
\hline 11 & $\mathbf{1 1}$ & 11 & 11 & \\
\hline 12 & $\mathbf{1 3}$ & 13 & 13 & \\
\hline
\end{tabular}




\subsection{Driving Power- Dependence Diagram}

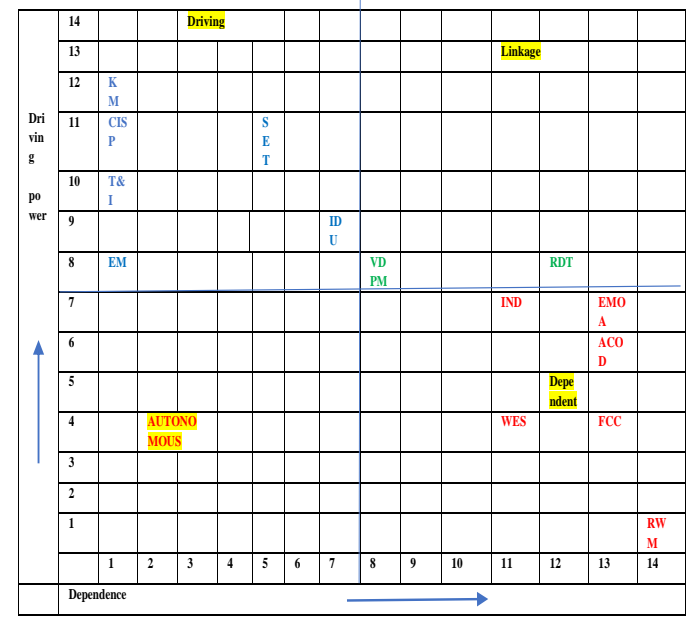

\subsection{ISM Diagraph}

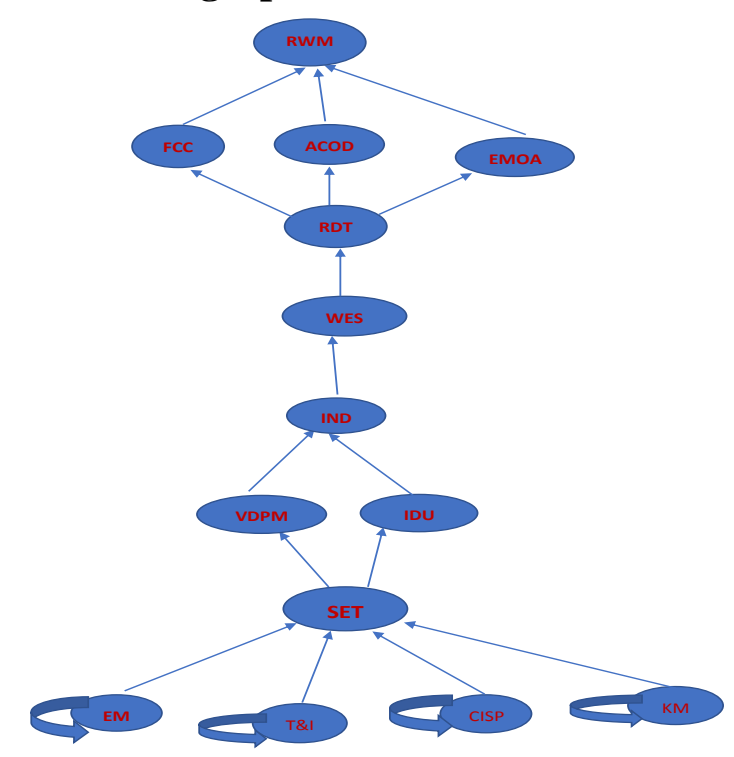

Fig 3.3.1 : ISM Diagraph

\subsection{Fuzzy ISM methodology}

\subsubsection{Formation of ISM based fuzzy MICMAC}

analysis model

With the help of key enablers this analysis is done so that drive the structure in different categories. However the relationship between the success factors may not be equal, some relation may be strong, especially strong and better. To rise above this problem of ISM, fuzzy MICMAC analysis is moved away as per following steps.

\subsubsection{Binary direct relationship matrix}

From the direct examination relationship between the enablers in the ISM, a binary direct reachability matrix (BDRM) is obtained and the diagonal entries are converted to zero. It has been observed that in the traditional MICMAC analysis the relationship is the only binary type, but in this paper fuzzy set theory (FST) is applied to enhance the responsiveness of MICMAC analysis. The BDRM is shown in Table XI. To convert from traditional MICMAC analysis to fuzzy MICMAC, a supplementary contribution of option of communication among the enablers is required. The supplementary contribution can be defined by qualitative consideration on 0-1 scale as shown in Table 3.4.1.

Table 3.4.1. Binary Direct Reachability Matrix

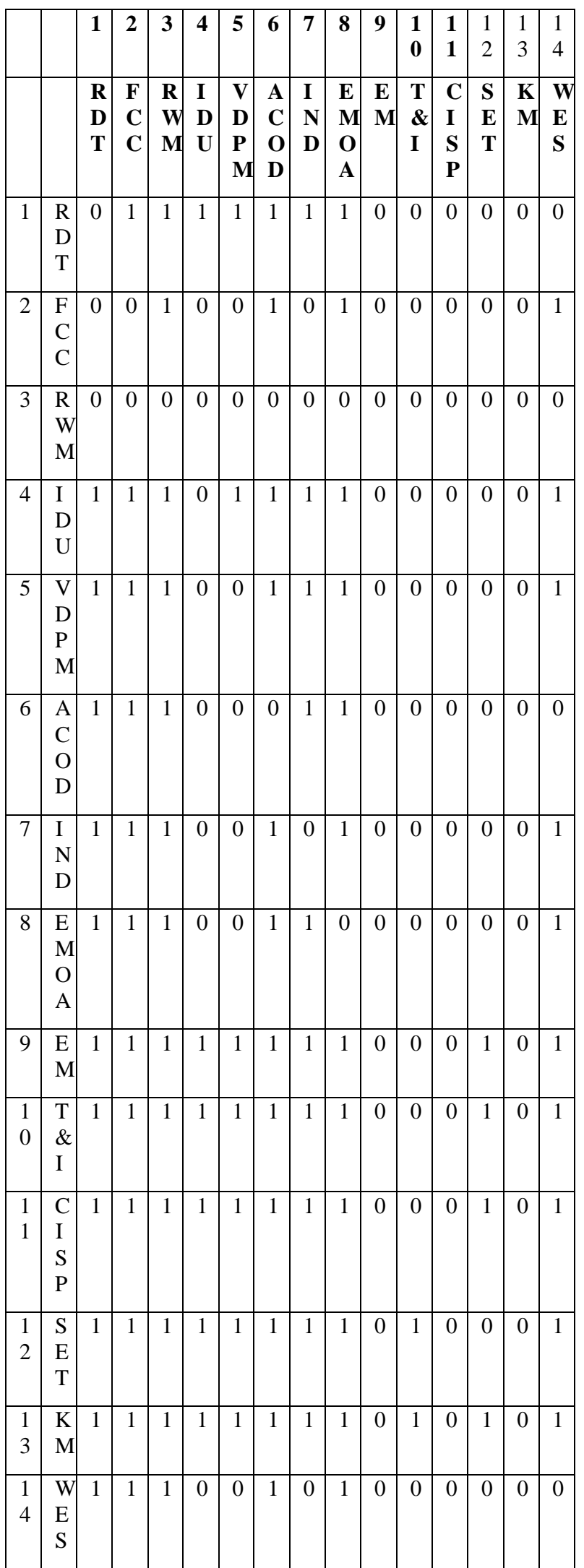


Table 3.4.2: Possibility of numerical values of reachability

\begin{tabular}{|c|c|c|c|c|c|c|c|}
\hline $\begin{array}{c}\text { Possibilit } \\
\mathbf{y} \text { of } \\
\text { reachabili } \\
\text { ty }\end{array}$ & No & $\begin{array}{c}\text { Ver } \\
\mathbf{y} \\
\text { low }\end{array}$ & low & $\begin{array}{c}\text { med } \\
\text { ium }\end{array}$ & $\begin{array}{c}\text { Hig } \\
\mathbf{h}\end{array}$ & $\begin{array}{c}\text { Ver } \\
\mathbf{y} \\
\text { high }\end{array}$ & $\begin{array}{c}\text { Co } \\
\mathbf{m p l} \\
\text { ete }\end{array}$ \\
\hline Value & 0 & 0.1 & 0.3 & 0.5 & 0.7 & 0.9 & 1 \\
\hline
\end{tabular}

\subsubsection{Fuzzy direct reachability matrix}

Table 3.4.3. Fuzzy Direct Reachability Matrix

\begin{tabular}{|c|c|c|c|c|c|c|c|c|c|c|c|c|c|c|c|c|}
\hline & & 1 & 2 & 3 & 4 & 5 & 6 & 7 & 8 & & 9 & \begin{tabular}{l|}
1 \\
$\mathbf{0}$
\end{tabular} & $\begin{array}{l}1 \\
1\end{array}$ & $\begin{array}{l}1 \\
2\end{array}$ & \begin{tabular}{|l|}
1 \\
3
\end{tabular} & $\begin{array}{l}1 \\
4\end{array}$ \\
\hline & & $\begin{array}{l}\text { R } \\
\text { D } \\
\text { T }\end{array}$ & $\begin{array}{l}\mathbf{F} \\
\mathbf{C} \\
\mathbf{C}\end{array}$ & $\begin{array}{l}\mathbf{R} \\
\mathbf{W} \\
\mathbf{M}\end{array}$ & $\begin{array}{l}\text { I } \\
\text { D } \\
\mathbf{U}\end{array}$ & $\begin{array}{l}\text { V } \\
\text { D } \\
\text { P } \\
\text { M }\end{array}$ & $\begin{array}{l}\text { A } \\
\mathbf{C} \\
\mathbf{O} \\
\mathbf{D}\end{array}$ & $\begin{array}{l}\text { I } \\
\mathbf{1} \\
\mathbf{D}\end{array}$ & & $\begin{array}{l}\mathbf{E} \\
\mathbf{M} \\
\mathbf{O} \\
\mathbf{A}\end{array}$ & $\begin{array}{l}\mathbf{E} \\
\mathbf{M}\end{array}$ & $\begin{array}{l}\mathbf{T} \\
\boldsymbol{\&} \\
\mathbf{I}\end{array}$ & $\begin{array}{l}\mathbf{C} \\
\mathbf{I} \\
\mathbf{S} \\
\mathbf{P}\end{array}$ & $\begin{array}{l}\mathbf{S} \\
\mathbf{E} \\
\mathbf{T}\end{array}$ & $\begin{array}{l}\mathbf{K} \\
\mathbf{M}\end{array}$ & $\begin{array}{l}\text { W } \\
\text { E } \\
\text { S }\end{array}$ \\
\hline 1 & $\begin{array}{l}\mathrm{R} \\
\mathrm{D} \\
\mathrm{T}\end{array}$ & 0 & 0 & $\dot{5}$ & $\dot{5}$ & 0 & 0 & 0 & & 0 & 0 & & 0 & 0 & 0 & 0 \\
\hline 2 & $\begin{array}{l}\mathrm{F} \\
\mathrm{C} \\
\mathrm{C}\end{array}$ & $\begin{array}{l}0 \\
\dot{5}\end{array}$ & 0 & $\dot{5}$ & 0 & 0 & 0 & 0 & & 0 & 0 & $\begin{array}{l}0 \\
. \\
5\end{array}$ & 0 & 0 & 0 & 0 \\
\hline 3 & $\begin{array}{l}\mathrm{R} \\
\mathrm{W} \\
\mathrm{M}\end{array}$ & $\begin{array}{l}0 \\
7\end{array}$ & $\begin{array}{l}0 \\
5\end{array}$ & 0 & $\begin{array}{l}0 \\
5\end{array}$ & 0 & 0 & 0 & & 0 & 0 & $\begin{array}{l}0 \\
. \\
5\end{array}$ & 0 & 0 & 0 & 0 \\
\hline 4 & $\begin{array}{l}\text { I } \\
\text { D } \\
U\end{array}$ & $\begin{array}{l}0 \\
5\end{array}$ & 0 & $\dot{5}$ & 0 & 0 & 0 & 0 & & 0 & 0 & & 0 & 0 & 0 & 0 \\
\hline 5 & $\begin{array}{l}\mathrm{V} \\
\mathrm{D} \\
\mathrm{P} \\
\mathrm{M}\end{array}$ & $\begin{array}{l}0 \\
5\end{array}$ & 0 & $\dot{5}$ & 0 & 0 & 0 & 0 & & 0 & 0 & & 0 & 0 & 0 & 0 \\
\hline 6 & $\begin{array}{l}\text { A } \\
\text { C } \\
\text { O } \\
\text { D }\end{array}$ & $\begin{array}{l}0 \\
6 \\
6\end{array}$ & $\begin{array}{l}0 \\
6\end{array}$ & $\begin{array}{l}0 \\
\dot{6}\end{array}$ & $\begin{array}{l}0 \\
\dot{6}\end{array}$ & $\begin{array}{l}0 \\
\dot{5}\end{array}$ & 0 & 0 & & 0 & 0 & $\begin{array}{l}0 \\
\dot{5}\end{array}$ & 0 & 0 & 0 & 0 \\
\hline 7 & $\begin{array}{l}\mathrm{I} \\
\mathrm{N} \\
\mathrm{D}\end{array}$ & 1 & 1 & 1 & 1 & 1 & 1 & 0 & & 0 & 0 & 1 & 1 & 1 & 1 & 1 \\
\hline 8 & $\begin{array}{c}\text { E } \\
M \\
O \\
A\end{array}$ & 1 & 1 & 1 & 1 & 1 & 1 & 1 & & 0 & 0 & 1 & 1 & 1 & 1 & 1 \\
\hline 9 & $\begin{array}{c}\mathrm{E} \\
\mathrm{M}\end{array}$ & 1 & 1 & 1 & 1 & 1 & 1 & 1 & & 1 & 0 & 1 & 1 & 1 & 1 & 1 \\
\hline $\begin{array}{l}1 \\
0\end{array}$ & $\begin{array}{l}\mathrm{T} \\
\& \\
\mathrm{I}\end{array}$ & 0 & 0 & 0 & 0 & 0 & 0 & 0 & & 0 & 0 & 0 & 0 & 0 & 0 & 0 \\
\hline $\begin{array}{l}1 \\
1\end{array}$ & \begin{tabular}{|c}
$\mathrm{C}$ \\
$\mathrm{IS}$ \\
$\mathrm{P}$
\end{tabular} & 1 & 1 & 1 & 1 & 1 & 1 & 0 & & 0 & 0 & 1 & 0 & 1 & 1 & 1 \\
\hline $\begin{array}{l}1 \\
2\end{array}$ & $\begin{array}{l}\mathrm{S} \\
\mathrm{E} \\
\mathrm{T}\end{array}$ & 1 & 1 & 1 & 1 & 1 & 1 & 0 & & 0 & 0 & 1 & 0 & 0 & 1 & 1 \\
\hline
\end{tabular}

\begin{tabular}{|l|l|l|l|l|l|l|l|l|l|l|l|l|l|l|l|}
\hline 1 & $\mathrm{~K}$ & 1 & 1 & 1 & 1 & 1 & 1 & 1 & 1 & 0 & 1 & 1 & 1 & 0 & 1 \\
3 & $\mathrm{M}$ & & & & & & & & & & & & & & \\
\hline 1 & $\mathrm{~W}$ & 1 & 1 & 1 & 1 & 1 & 1 & 1 & 1 & 0 & 1 & 1 & 1 & 1 & 0 \\
4 & $\mathrm{E}$ & & & & & & & & & & & & & & \\
& $\mathrm{S}$ & & & & & & & & & & & & & & \\
\hline
\end{tabular}

\subsubsection{Fuzzy Mic-Mac stabilized matrix}

After the formation of fuzzy direct relationship matrix (FDRM) as shown in Table XIII to find the relationship between MCEs, take FDRM as an initial table and further process is starting. Apart from that multiplication of matrix is repeated until the hierarchies of the driving and dependence power constant. Fuzzy matrix multiplication is based on Boolean matrix multiplication (Kandasamy et al., 2007). According, to fuzzy set theory, when two fuzzy matrices are multiplied the product matrix is also fuzzy matrix.

\section{CONCLUSIONS AND FUTURE RESEARCH}

Many traditional industries are unable to face the pressure of competition. Only through adoption of new technology traditional industries can survive. While designing solutions, social, economic, organizational and technological aspects need to be considered. ICT platforms will be accepted if there are social and organizational readiness and economic viability built into the project concept. The present research made use of ISM methodology to recognize the inter-relationship amongst the various barriers and success factors to carpet weaving and textile industry . Other techniques such as fuzzy DEMAIC and ISM - SEM based methodology can also be used .

\section{REFERENCES}

[1] Warfield, J.N. 1974. Developing interconnection matrices in structural modelling. In the proceedings of IEEE Transactions on System, Man, and Cybernetics (SMC), 4 (1), 81-87.

[2] Mamidipudi , A. and Bijker, W.E. 2018. Innovation in Indian Handloom Weaving, Technology and Culture 59(3), 509-545 . DOI: 10.1353/tech.2018.0058.

[3] Bowonder, B. Swamy, J. and Mastakar, N. 2005. Regaining Competitiveness using an ICT platform in a Traditional Industry: Adoption of Computer Aided Design for Carpet Weaving. available at http://inderscience.metapress.com/content/ ep4x7x8a6wmcab7q/.

[4] Darbanhosseiniamirkhiz, M., Shabanifar, S., Ismail, W.K.W. 2011. Adoption of Technology in the Hand Woven Carpet Industry: The Case of Alinasab Carpet Company, Australian Journal of Basic and Applied Sciences, 10, 551-559.

[5] Das, P.K.' Shukla, K.P.' and Oryx, F.G., 1992. An Occupational Health Programme for Adults and Children in the Carpet Weaving Industry, Mirzapur, India: A Case Study in the Informal Sector. Sm. Sci. Med., Vol. 35, No. 10, pp-1293-1302.

[6] D. B. Yoffi 1996. Competing in the Age of Digital Convergence, California Management Review, Vol. 38, No. 4, 1996. 